\title{
Modelling Aspects of Tumour Metabolism
}

Maini, Philip K and Gatenby, Robert A and Smallbone, Kieran

2010

MIMS EPrint: 2010.45

Manchester Institute for Mathematical Sciences

School of Mathematics

The University of Manchester

\footnotetext{
Reports available from: http://eprints.maths.manchester.ac.uk/

And by contacting: The MIMS Secretary

School of Mathematics

The University of Manchester

Manchester, M13 9PL, UK
} 


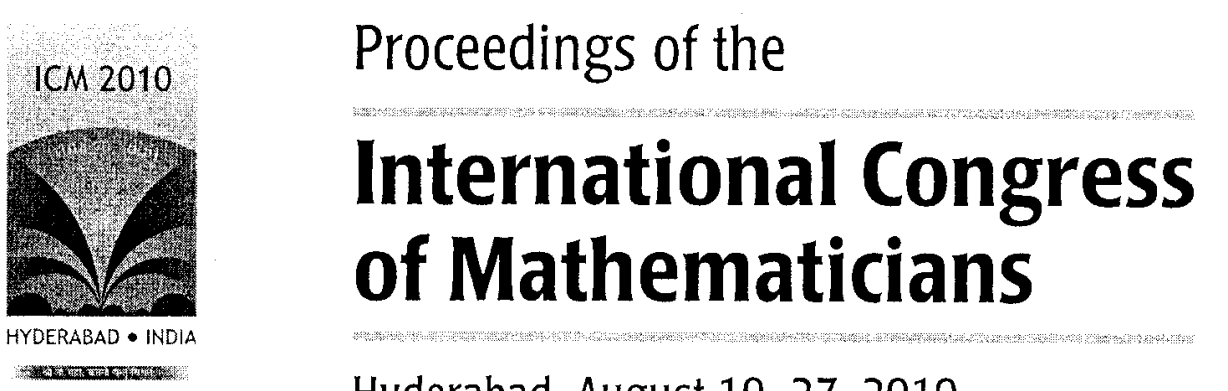

Hyderabad, August 19-27, 2010

\section{Volume IV}

\section{Invited Lectures}

Editor

Rajendra Bhatia

Co-Editors

Arup Pal

G. Rangarajan

V. Srinivas

M. Vanninathan

Technical Editor

Pablo Gastesi 
Editor

Rajendra Bhatia, Indian Statistical Institute, Delhi

\section{Co-editors}

Arup Pal, Indian Statistical Institute, Delhi

G. Rangarajan, Indian Institute of Science, Bangalore

V. Srinivas, Tata Institute of Fundamental Research, Mumbai

M. Vanninathan, Tata Institute of Fundamental Research, Bangalore

\section{Technical editor}

Pablo Gastesi, Tata Institute of Fundamental Research, Mumbai

\section{Published by}

Hindustan Book Agency (India)

P 19, Green Park Extension

New Delhi 110016

India

email: info@hindbook.com

http://www.hindbook.com

\section{Copyright $\odot 2010$, Authors of individual articles.}

No part of the material protected by this copyright notice may be reproduced or utilized in any form or by any means, electronic or mechanical, including photocopying, recording or by any information storage and retrieval system, without written permission from the copyright owner information storage and retrieval system, without written permission from the copyright owner,
who has also the sole right to grant licences for translation into other languages and publication thereof.

\section{ISBN 978-81-85931-08-3}

Exclusive distribution worldwide except India

World Scientific Publishing Co. Pte. Ltd.

5 Toh Tuck Link, Singapore 596224 


\section{Modelling Aspects of Tumour Metabolism}

Philip K. Maini*, Robert A. Gatenby ${ }^{\dagger}$, and Kieran Smallbone ${ }^{\ddagger}$

\section{Abstract}

We use a range of mathematical modelling techniques to explore the acidmediated tumour invasion hypothesis. The models make a number of predictions which are experimentally verified. The therapeutic implications, namely either buffering acid or manipulating the phenotypic selection process, are described.

Mathematics Subject Classification (2010). 92C50

Keywords. Carcinogenesis - Glycolytic phenotype - Mathematical modelling

\section{Biological Background}

Cancer cell populations are extremely heterogeneous, displaying a wide range of genotypic and phenotypic differences [7]. For example, studies of clinical breast cancers have shown that every cell line exhibited a novel genotype, meaning no prototypic cancer cell can be defined. It is likely that several of the lethal phenotypic traits of cancer are not the direct result of genetic changes, but rather arise from the unique physiological environments of tumours.

The tumour microenvironment is significantly different from that of normal tissue. Marked fluctuations can be seen in glucose, lactate, acidic $\mathrm{pH}$ and

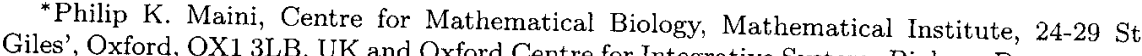
Giles', Oxford, OX1 3LB, UK and Oxford Centre for Integrative Systems Biology, Department Biochemstry, South Parks Road, Oxford OX1 3QU. E-mail: maini@maths.ox.ac.uk.

USA. E-mail: robert.gatenby@moffitt.or Center, 12902 Magnolia Drive, Tampa, FL 33612, U. E-mail: robert.gatenby@moffitt.org.

FKieran Smallbone, Manchester Centre for Integrative Systems Biology, Manchester Interdisciplinary Biocentre, 131 Princess Street, Manchester, M1 7DN, UK.

-mail: kieran.smallbone@manchester.ac.uk.

PKM was partially supported by a Royal Society-Wolfson Merit award. PKM and RAG were partially supported by NIH grant 1U54CA143970-01. KS acknowledges the support of the BBSRC/EPSRC grant BB/C008219/1 "The Manchester Centre for Integrative Systems Biology (MCISB) 
oxygen tensions. These variations have their roots both in poor perfusion and metabolic changes. The chaotic vasculature of tumours creates an unbalanced blood supply. As a consequence, many regions within tumours are found to be transiently or chronically hypoxic (poorly oxygenated). Cells respond to periods of hypoxia by converting to anaerobic metabolism, or glycolysis, which in turn produces lactic acid and brings about lower tissue $\mathrm{pH}$. However, the pioneering work of Warburg [22] showed that tumour acidification can occur independently of hypoxia. The increased reliance on glycolysis to produce energy in many aggressive tumours occurs even in the presence of sufficient oxygen. Thus acidification is an intrinsic property of both poor vasculature and altered tumour cell metabolism.

The constitutive adoption of increased aerobic glycolysis is known as the glycolytic phenotype. The inefficiency of this anaerobic metabolism is compensated for through a several-fold increase in cellular glucose consumption. This phenomenon is now routinely exploited for tumour imaging through fluorodeoxyglucose positron emission tomography (FDG-PET). PET has confirmed that the vast majority $(>90 \%$ ) of human primary and metastatic tumours demonstrate increased glucose uptake indicating abnormal metabolism. Furthermore. PET has been used to show a direct correlation between tumour aggressiveness and the rate of glucose consumption [4].

The presence of the glycolytic phenotype in the malignant phenotype of such a wide range of cancers seems inconsistent with an evolutionary model of carcinogenesis. Due to the Darwinian dynamics at play, it is reasonable to assume the common appearance of a specific phenotype within a large number of different cancer populations is evidence that it must confer a significant growth advantage. However, the proliferative advantages gained from altered glucose metabolism are far from clear. Firstly, anaerobic metabolism is more than an order of magnitude less efficient than its acrobic countcrpart, producing only 2 ATP molecules per glucose molecule in comparison to approximately 36 ATP molecules. Secondly, the hydrogen ions produced as a result of giycolysis cause a consistent acidification of the extracellular space that is toxic [17]. Intuitively, one would expect the Darwinian forces prevailing during carcinogenesis to select against this inefficient and environmentally toxic phenotype, in favour of more optimal metabolic regimes.

Gatenby and Gillies [11] propose that evolution of aerobic glycolysis is the result of environmental constraints imposed by the morphology of the ducts in which premalignant lesions evolve. Initially, normal epithelial cells grow along a basement membrane, with the epithelial layer at most a few cells thick. Homeostasis mechanisms do not normally allow growth of these cells away from the basement membrane. However, following initial genetic events in the carcinogenesis pathways such as those depicted by the Fearon-Vogelstein model [6], the cells become hyperplastic, leading to a thickening of the epithelial layer, pushing cells into the lumen and away from the membrane. Since the blood vessels remain outside the basement membrane, nutrients and waste must diffuse over longer and longer distances. As a result, it is likely that hyperplastic cells beyond the Thomlinson-Gray limit of 100-150 $\mu \mathrm{m}$ [21] from the basement membrane will experience profound hypoxia, which will initiate a sequence of critical cellular adaptations and environmental changes. Specifically, it is proposed that hypoxia leads to constitutive upregulation of glycolysis which, in turn, results in increased $\mathrm{H}^{+}$production and acidification of the microenvironment. This decreased extracellular $\mathrm{pH}\left(\mathrm{pH}_{\mathrm{X}}\right)$ is toxic to the local populations, in turn selecting for cells that are resistant to acid-induced toxicity. Acidosis also selects for motile cells that eventually breach the basement membrane, gaining access to existing and newly formed blood and lymphatic routes for metastasis.

Gatenby and Gawlinski [10] point out that the tumour phenotype that emerges from the sequence above, constitutively increasing acid production and becoming resistant to acid-induced toxicity, has a powerful growth advantage over its normal counterparts. They propose that acidity may play a key role in mediating tumour invasion. The key idea is that the transformed tumour metabolism with increased use of glycolysis and acid secretion alters the microenvironment by substantially reducing tumour extracellular $\mathrm{pH}$, usually by more than $0.5 \mathrm{pH}$ units. The $\mathrm{H}^{+}$ions produced by the tumour then diffuse along concentration gradients into the adjacent normal tissue. This acidification leads to death of normal cells; tumour cells, however, are relatively resistant to acidic $\mathrm{pH}_{\mathrm{X}}$. Whilst normal cells die in environments with a persistent $\mathrm{pH}$ below about 7 , tumour cells typically exhibit a maximum proliferation rate in a relatively acidic medium ( $\mathrm{pH} 6.8$ ) [3]. As a result, the tumour edge can be seen as forming a travelling wave progressing into normal tissue, preceded by another travelling wave of increased microenvironmental acidity.

\section{Continuum Modelling Approaches}

Population ecology methods provide a means for examining tumours, not as an isolated collection of transformed cells, but rather as an invading species in a previously stable multicellular population. Gatenby and Gawlinski [10] model the tumour-host interface as a network of interacting normal and malignant cell populations, using coupled, non-linear differential equations. The interactions are then explored to define the crucial parameters that control tumourigenesis and to demonstrate the limitations of traditional therapeutic strategies.

Tumour cell populations, as with any invading population in biology, must directly perturb their environment in such a way as to facilitate their own growth while inhibiting the growth of the original community. The commonality of altered tumour metabolism, in particular the adoption of the glycolytic phenotype in most cancers, led Gatenby and Gawlinski to propose the acidmediated tumour invasion hypothesis outlined above. The authors propose that tumour cells' increased acid secretion, coupled with their resistance to low extracellular $\mathrm{pH}$, may provide a simple but complete mechanism for cancer invasion. 
The hypothesis is modelled as a system of three coupled partial differential equations (PDEs), determining the spatio-temporal distribution of three fields the normal tissue density $N_{1}$, the tumour tissue density $N_{2}$, and the concentration of excess hydrogen ions $L$. The model includes: (1) logistic cellular growth (2) normal cell death due to exposure to acid; (3) acid production by tumour cells; (4) acid reabsorption and buffering; and (5) spatial diffusion of acid and cells. It takes the form

$$
\begin{aligned}
\frac{\partial N_{1}}{\partial t} & =r_{1} N_{1}\left(1-\frac{N_{1}}{K_{1}}\right)-d_{1} L N_{1} \\
\frac{\partial N_{2}}{\partial t} & =r_{2} N_{2}\left(1-\frac{N_{2}}{K_{2}}\right)+D_{2} \nabla \cdot\left[\left(1-\frac{N_{1}}{K_{1}}\right) \nabla N_{2}\right] \\
\frac{\partial L}{\partial t} & =r_{3} N_{2}-d_{3} L+D_{3} \nabla^{2} L
\end{aligned}
$$

where $r_{1}$ and $r_{2}$ are the growth rates of the normal and tumour cell populations, respectively, $K_{1}$ and $K_{2}$ their carrying capacities, $D_{2}$ scales the diffusion coefficient for tumour cells, $d_{1}$ is the normal cell susceptibility to acid, $r_{3}$ the rate of hydrogen ion production by tumour cells, $d_{3}$ the combined rate of acid removal by blood vessels and buffering, and $D_{3}$ the diffusion coefficient for hydrogen ions in tissue. Notice that there is no normal cell diffusion within the model, in recognition of the fact that healthy tissue is well-regulated and participating normally in an organ. Notice also that the tumour diffusion coefficient is constructed such that when normal tissue is at its carrying capacity, the diffusion coefficient for tumour tissue is zero and the tumour is confined. This final assumption is at the heart of the model: tumour tissue is unable to spread without first diminishing the surrounding healthy tissue from its carrying capacity.

In non-dimensional form, Eqs. (1)-(3) become

$$
\begin{aligned}
& \frac{\partial \eta_{1}}{\partial \tau}=\eta_{1}\left(1-\eta_{1}\right)-\delta_{1} \Lambda \eta_{1}, \\
& \frac{\partial \eta_{2}}{\partial \tau}=\rho_{2} \eta_{2}\left(1-\eta_{2}\right)+\Delta_{2} \nabla_{\xi} \cdot\left[\left(1-\eta_{1}\right) \nabla_{\xi} \eta_{2}\right], \\
& \frac{\partial \Lambda}{\partial \tau}=\delta_{3}\left(\eta_{2}-\Lambda\right)+\nabla_{\xi}^{2} \Lambda .
\end{aligned}
$$

The system has four spatially-homogeneous steady states:

- $\eta_{1}=0, \eta_{2}=0$ : the trivial solution.

- $\eta_{1}=1, \eta_{2}=0$ : corresponding to normal healthy tissue with no tumour cells present.

- $\eta_{1}=1-\delta_{1}, \eta_{2}=1$ : corresponding to tissue consisting of both normal and tumour cells at an intermediate level, which may be interpreted as a less aggressive (but invasive) tumour. (Note that this is only biologically realistic for non-negative values of the density).

- $\eta_{1}=0, \eta_{2}=1$ : corresponding to total tumour invasion.
Linear stability analysis [15] shows us that the trivial state and the state corresponding to normal cells alone are unconditionally unstable. Both the invasive state and the coexisting state are conditionally, but mutually exclusively, stable. The critical parameter is found to be $\delta_{1}=d_{1} r_{3} K_{2} / d_{3} r_{1}$. Depending on the value of this dimensionless parameter, either the steady state for total destruction of normal tissue $\left(\delta_{1}>1\right)$ or the steady state with the tumour and normal cells coexisting $\left(\delta_{1}<1\right)$ is stable. Thus as the value of $\delta_{1}$ passes through the critical value of 1 , the entire system will change from a less aggressive to a more aggressive invasive pattern. For example, increased tumour vascularity will increase $K_{2}$ and push the system to an unstable steady state. A detailed analytical study of this system reveals a rich variety of wave propagation dynamics with fast and slow waves [5].

Late-time travelling wave solutions [15] to Eqs. (4)-(6) are computed in Fig. 1. The first point of note is that the model predicts a smooth $\mathrm{pH}$ gradient extending from the tumour edge into the peritumoural tissue. The authors reanalyse data presented by Martin and Jain [12] relating to in vivo interstitial $\mathrm{pH}$ profiles for the VX2 rabbit carcinoma and its surrounding normal tissue, demonstrating that the data are consistent with the presence and approximate range of the $\mathrm{pH}$ gradient predicted by the model. Most significantly, however the model predicts that (when $\delta_{1}>1$ ) there exists a previously unrecognised acellular gap separating the advancing tumour and receding host tissue fronts. In subsequent in vitro experiments, the authors found that, of 21 specimens of human squamous cell carcinoma of the head and neck, 14 were judged to show such a gap. Naked nuclei and morphologically disrupted cells were frequently observed scattered within the gap, or at its edge, as predicted by the model [10].

The GG model focuses on malignant invasion and not transition from benign to malignant states. This issue is addressed in $[19,20]$, in a model in which this transition occurs as a critical parameter breaches a bifurcation value. This is consistent with data [8] showing that the acquisition of the angiogenic phenotype radically and abruptly alters the tumour growth pattern from non-invasive, slow growth to rapidly expanding, invasive growth.

\section{Hybrid Modelling Approaches}

Despite the apparent success of Gatenby and Gawlinski's model in examining large, clinically apparent tumours, its relevance to early tumour growth is not clear. Continuous partial differential equation models are well suited to modelling large populations, but individual-based models such as cellular automata $(\mathrm{CA})$ are more appropriate when the evolutionary dynamics of individual cells must be considered. However, traditional CA methods lack the ability to deal with continuously varying elements such as substrate diffusion and utilisation. Thus, hybrid CA have been developed to investigate early cancer development $[2,16]$. 


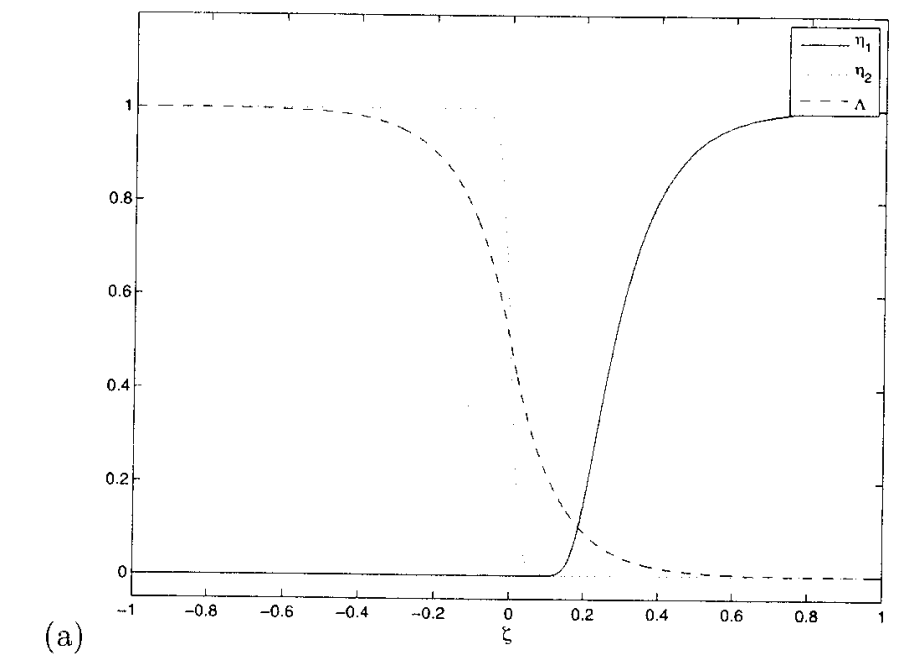

(a)

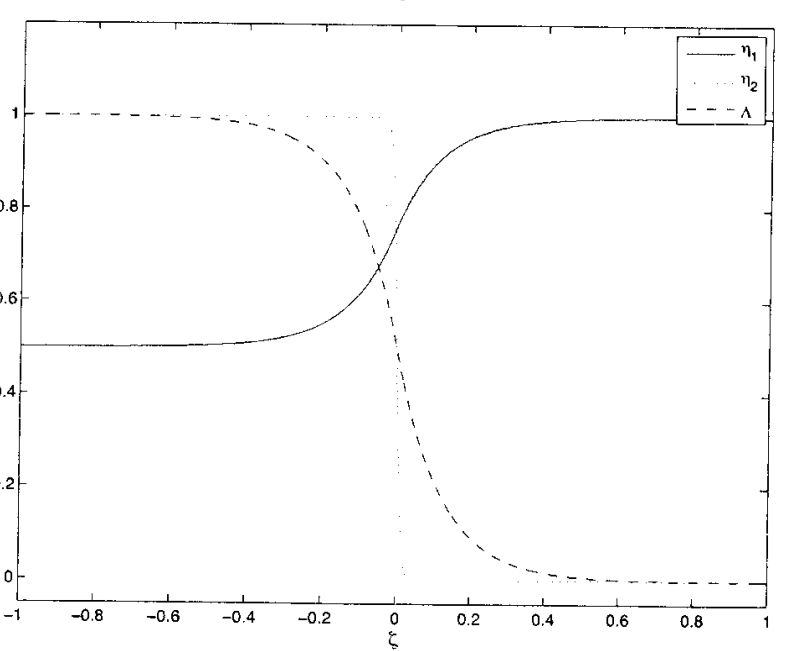

The cellular automaton model used here [18] is composed of an $M \times N$ array of automaton elements with a specific rule-set governing their evolution, as well as glucose $(g)$, oxygen $(c)$ and $\mathrm{H}^{+}(h)$ fields, each satisfying reaction-diffusion equations. A two-dimensional automaton is used as we focus on growth away from the basement membrane, rather than along the duct. In the model we reflect the avascular geometry of premalignant epithelia by assuming that one edge of the array represents the basement membrane.

We consider the selective pressures placed on a number of different possible tumour phenotypes. Initially, the automaton consists of a single layer of normal epithelial tissue. As well as proliferation and death, these cells may randomly undergo three possible heritable changes, either through mutations or epigenetic changes such as alterations in the methylation patterns of promoters. The cells may become hyperplastic (allowing growth away from the basement membrane), glycolytic (increasing their rate of glucose uptake and utilisation) or acid-resistant (requiring a lower extracellular $\mathrm{pH}$ to induce toxicity). These three changes give rise to $2^{3}=8$ different phenotype combinations, and thus eight competing cellular populations.

Cellular metabolism Suppose that a cell consumes glucose and oxygen at rates $\phi_{g}$ and $\phi_{c}$, respectively, and that they are used to produce ATP and $\mathrm{H}^{+}$ at rates $\phi_{a}$ and $\phi_{h}$. In non-dimensional form, we have

$$
\begin{aligned}
& \phi_{g}= \begin{cases}g & \text { in a normal cell } \\
k g & \text { in a glycolytic cell }\end{cases} \\
& \phi_{c}=c, \\
& \phi_{a}=c+n\left(\phi_{g}-c\right) \\
& \phi_{h}=\phi_{g}-c,
\end{aligned}
$$

subject to the condition $\phi_{g} \geq c$.

Metabolite profiles After each automaton generation, the known rates of metabolite consumption and production for each cell are used to calculate the corresponding metabolite profiles. Note that metabolite diffusion time-scales ( $\sim$ minutes) are much shorter than the cellular proliferation time-scale ( $\sim$ days), and thus we may assume that metabolites are in equilibrium at all times. Assuming that diffusion is the primary method for metabolite movement within the tissue, profiles are given in non-dimensional form by

$$
\begin{aligned}
d_{g}^{2} \nabla_{\xi}^{2} g & =\phi_{g} \\
d_{c}^{2} \nabla_{\xi}^{2} c & =\phi_{c} \\
\nabla_{\xi}^{2} h & =-\phi_{h}
\end{aligned}
$$


which may be solved on the square grid using a finite difference approximation. As boundary conditions, we assume zero flux at the edge furthest from the basement membrane (as there are no sources or sinks beyond this point), and periodic boundary conditions at the two sides. At the membrane, we assume glucose and oxygen are fixed at their normal levels $g_{0, j}=c_{0, j}=1$ (as the stroma is well-vascularised); $\mathrm{H}^{+}$is also fixed, $h_{0, j}=h_{X}$, where the parameter $h_{X}$ reflects the level of systemic acidosis.

Cell dynamics Cells may proliferate, adapt or die, and cells with different phenotypic patterns respond to the microenvironmental pressures in different ways. As such, competition is incorporated into the model: for a new population to progress and grow, it must successfully compete for space and resources with existing populations. The rules governing the evolution of the automaton elements are as follows:

- If the amount of ATP produced by a cell $\left(\phi_{a}\right)$ falls below a critical threshold value, $a_{0}$, it dies, and the element becomes empty; $a_{0}$ represents the level of ATP required for normal cellular maintenance.

- The local $\mathrm{H}^{+}$level may also induce cellular death, with probability $p_{\text {dea }}$ defined by

$$
p_{\text {dea }}= \begin{cases}h / h_{N} & \text { in a normal cell } \\ h / h_{T} & \text { in an acid-resistant cell }\end{cases}
$$

where $h_{N}<h_{T}$. Thus the probability of cell death increases with acidity and the cell will always die if the $\mathrm{H}^{+}$level is greater than $h_{N}$ or $h_{T}$, dependent on the cell type under consideration.

- If the cell is not attached to the basement membrane, and is not hyperplastic, it dies.

- If the cell does not die through any of the mechanisms above, it either attempts to divide, with probability $p_{\text {div }}$, or becomes quiescent. The probability of division is a function of the cellular ATP production

$$
p_{\text {div }}=\left(\phi_{a}-a_{0}\right) /\left(1-a_{0}\right) .
$$

Hence we assume that the probability of division is proportional to the ATP generated that is not needed for maintenance. If there is more than one neighbouring empty space, the new cell goes to the element with the largest oxygen concentration (following [1]).

- If a cell divides, each of the two daughter cells has probability $p_{\mathbf{a}}$ of randomly acquiring one of the three heritable characteristics (hyperplasia glycolysis and acid-resistance). In order to avoid bias in the model, we assume these changes are reversible. For example, a cell displaying constitutive up-regulation of glycolysis may revert to normal glucose metabolism if this metabolism is most appropriate for the current microenvironmental conditions, the cell will successfully compete for resources with its neighbours.

Fig. 2 presents a typical result from our hybrid CA model. Initially, normal epithelial cells line the basement membrane (a). Acquisition of the hyperplastic phenotype allows growth away from the membrane towards the oxygen diffusion limit (b). Beyond this point, cells cannot exist as the oxygen levels are insufficient to meet cellular ATP demands. This drives adaptation to a glycolytic phenotype, less reliant on oxygen for ATP production (c). The increased ATP levels within glycolytic cells give a competitive advantage over the existing population, thus glycolytic cells dominate the system. Note, however, that the total number of cells within the system has decreased; the increased reliance on glycolysis has resulted in higher levels of acidity, in turn inducing cell death. Further adaptation occurs to an acid-resistant phenotype (d). Increased use of glycolysis allows growth well beyond the oxygen diffusion limit, whilst the cells are more resistant to the resulting acidosis. (a)

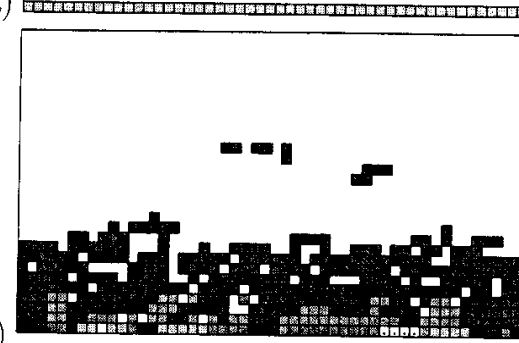

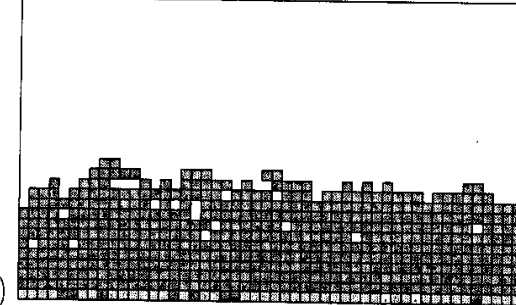

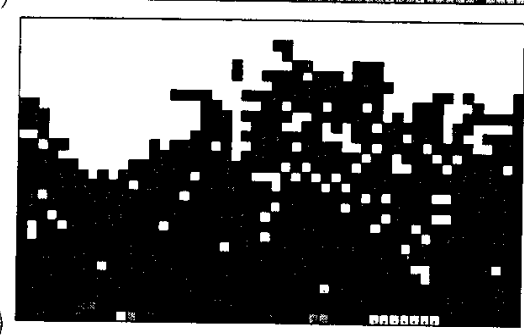

Figure 2. The temporal evolution of a typical cellular automaton after (a) $t=0$, (b) $t=100$, (c) $t=250$ and (d) $t=300$ generations. Darker denotes a more aggressive phenotype. Shown are normal epithelial (w), hyperplastic ( hyperplastic-glycolytic ( ) and hyperplastic-glycolytic-acid-resistant ( $\mathbf{m})$ cells. Cells with other phenotypic patterns are shown as $\square$. Parameter values used are $N=50, n=5.6 \times 10^{-2}, k=10$, $d_{g}=1.3 \times 10^{2}, d_{c}=5, a_{0}=0.1, h_{N}=9.3 \times 10^{2}, h_{T}=8.6 \times 10^{3}, p_{\mathrm{a}}=10^{-3}$ and $h_{X}=0$ 
It is interesting to note that throughout the simulations performed, the heritable changes within the dominant population are accumulated in this same order. Within our model, the underlying environmental selection parameters drive the cells to always follow this adaptive pathway - escaping in turn from the constraints of limited proliferation (hyperplasia), substrate availability (glycolysis) and waste removal (acid-resistance). The same order of progression occurs despite allowing phenotypic reversibility within our model. This means mutations are not a necessary mechanism for phenotypic variation within tumour tissue; rather the model demonstrates that reversible, epigenetic changes are sufficient to drive global change. Of course reversibility in not necessary to observe this adaptation; if irreversible, we would see the same phenotype emerge on a slightly shorter time-scale.

\section{Potential Applications I: Bicarbonate Treatment}

Recently, we have used compartmental models to predict the effect of bicarbonate treatment on humans and shown, through a sensitivity analysis, that this could best be made more effective by combination with proton inhibitors [13].

\section{Potential Applications II: Exercise}

There is accumulating evidence that regular physical activity is an effective cancer prevention strategy. Friedenrich and Orenstein [9] recently reviewed over 170 epidemiological studies and concluded that evidence for decreased cancer risk with increased physical activity was convincing for breast and colon cancer, probable for prostate cancer and possible for lung cancer. We hypothesise that exercise produces toxicity within in situ cancers through transient decreases in serum $\mathrm{pH}$ and, by doing so, will also transiently cause significant further decrease in extracellular $\mathrm{pH}$ in the already acidic regions within in situ cancers. This abrupt increase in acid concentrations will result in tumour cell death and interrupt the adaptive mechanisms necessary for subsequent evolution to the malignant phenotype. To test the hypothesis, we extend the CA to include variations in systemic $\mathrm{pH}$.

When investigating transient acidosis, each time-step is split into two parts: a proportion of time $\tau \in[0,1]$ spent at high acidity $h_{X} \gg 1$, followed by a proportion of time $1-\tau$ at normal acidity $h_{X}=0$. Letting $p_{0}$ denote the probability of death $p_{\text {dea }}$, division $p_{\text {div }}$, or mutation $p_{\mathrm{a}}$ during one time unit (as defined previously), the corresponding probability $p$ of occurrence during the acidic phase is given by

$$
p(\tau)=1-\left(1-p_{0}\right)^{\tau},
$$

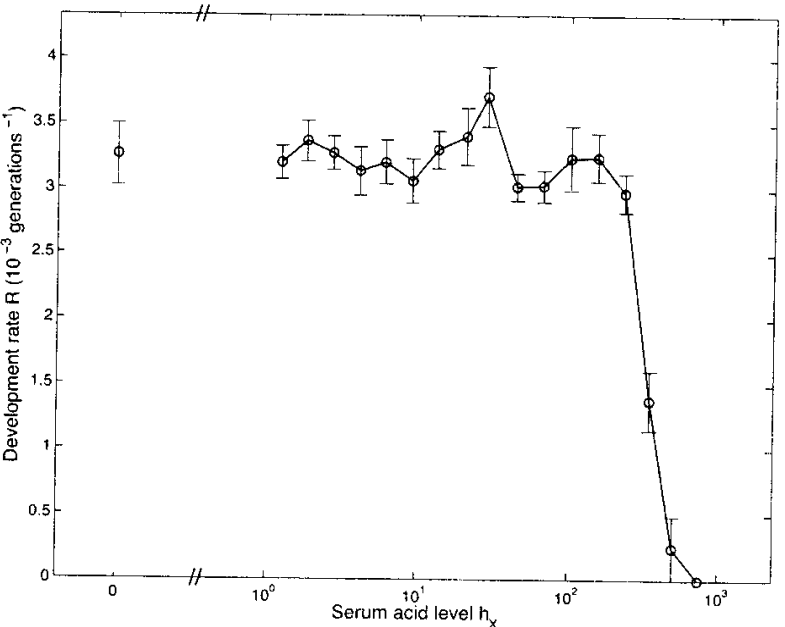

(a)

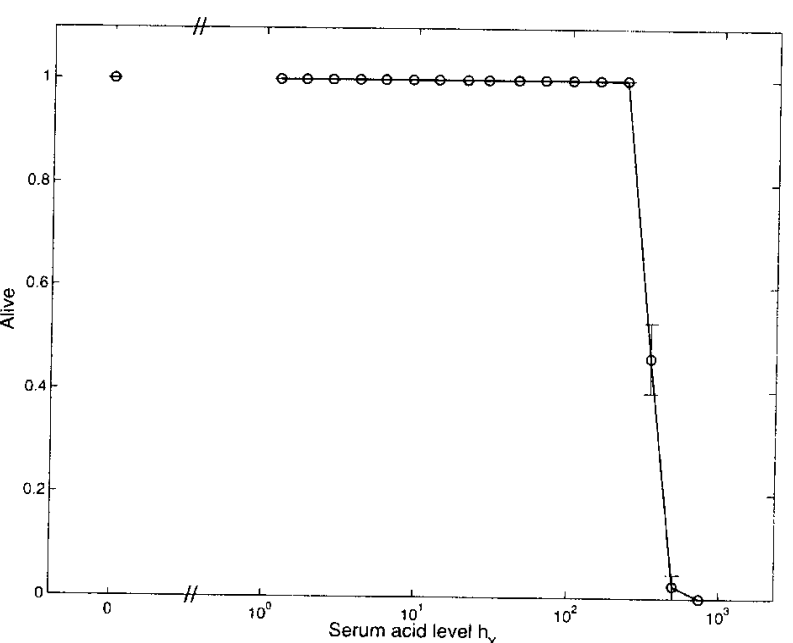

Figure 3. Effect of sustained acidosis. (a) Variation in the development rate $R$ with serum acid level $h_{X}$ (plotted on a log scale). Each data point is the mean value of $R$ calculated over 50 simulations, whilst the accompanying error bars show the standard errors of these means. (b) Variation in epithelium survival with $h_{X}$.

whilst the probability of occurrence during the normal phase is given by

$$
p(1-\tau)=1-\left(1-p_{0}\right)^{1-\tau}
$$

In order to examine the effects of parameter changes on system dynamics, we define a measure of the 'fitness' of a specific parameter set. Let 'invasive' 
(a)
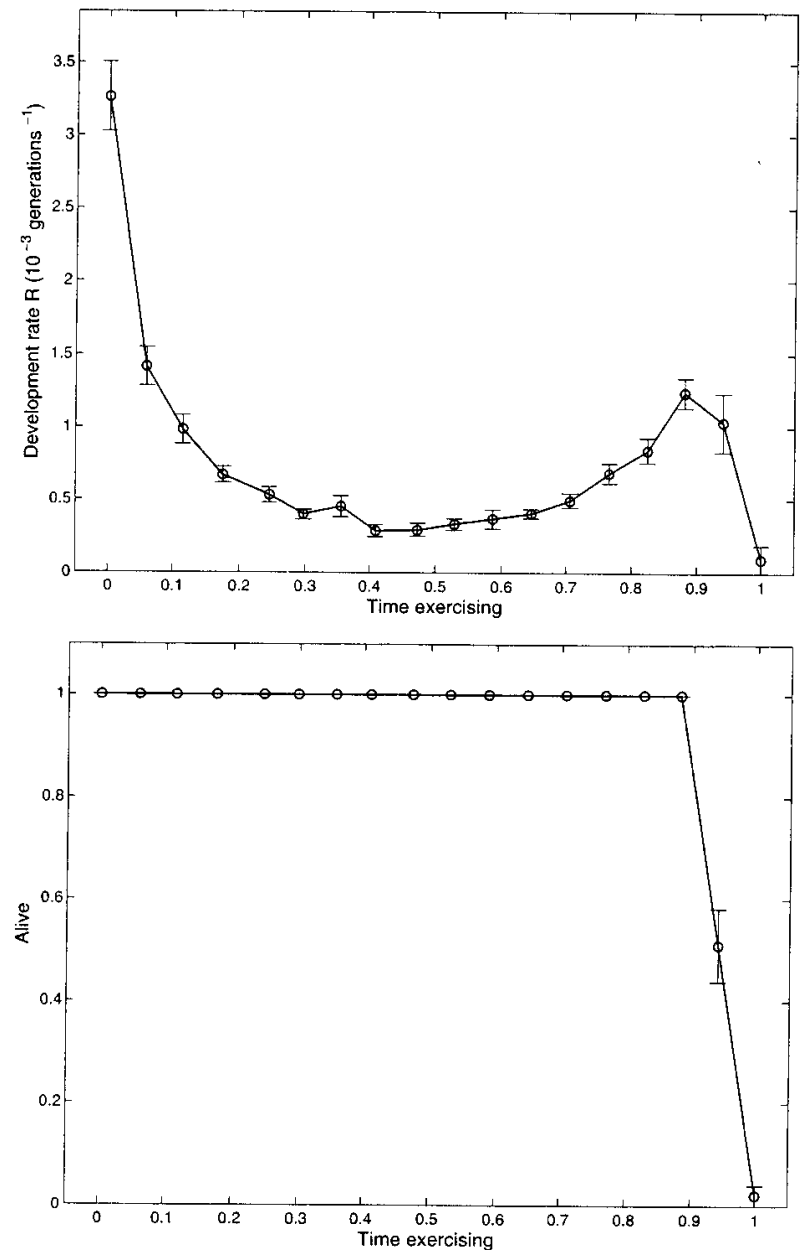

(b)

Figure 4. Effect of transient acidosis. (a) Variation in the development rate $R$ with proportion of time under exercise. Exercise is assumed to correspond to high acidity $\left(h_{X}=400\right)$, whilst during rest acidity drops to normal levels $\left(h_{X}=0\right)$. (b) Variation in epithelium survival with exercise time.

be used to describe cells displaying all three heritable changes - hyperplasia, glycolysis and acid resistance. For a particular automaton, let $T$ denote the number of generations after which $95 \%$ of the cells in the system display the invasive phenotype; thus $T$ is representative of the amount of time taken for full carcinogenesis to occur. Now let the development rate $R=T^{-1}$, where we take $R=0$ if $T \geq 5000$ (equivalent to approximately 20 years) - i.e. assume no carcinogenesis occurs. Automata with a higher value of $R$ proceed more quickly through the carcinogenesis pathway.

From Fig. 3 (a) we see how the development rate $R$ varies with changes in serum acidity $h_{X}$. We vary the external acid levels from $h_{X}=0$ (normal) to $h_{X} \sim 1000$, equivalent to $\mathrm{pH} \mathrm{6.8,} \mathrm{corresponding} \mathrm{to} \mathrm{the} \mathrm{threshold} \mathrm{for} \mathrm{normal}$ cell survival [16]. Development rate $R$ remains fairly constant until $h \sim 100$ (a drop of around $0.1 \mathrm{pH}$ units), when a marked decrease is observed. Looking further however, we see (Fig. 3 (b)) that this result follows simply because the harsher conditions lead to death of the entire epithelium; normal cells die out before having the opportunity to turn cancerous.

Since the model predicts that permanent acidosis cannot arrest cancer development, we move on to investigate transient acidosis, allowing the system to spend a certain proportion of time at high acidity and a certain proportion at normal acidity; this transient acidosis mimics what occurs when engaging in rigorous exercise followed by rest. In Fig. 4 (a) we see how the development rate $R$ varies with the amount of time exercising. We see that only a small proportion of time spent at low $\mathrm{pH}(h=400$, a drop of around $0.25 \mathrm{pH}$ units) leads to a significant reduction in $R$. By contrast to the previous figure, the behaviour is not due to total epithelial death (Fig. 4 (b))

\section{Discussion}

We have used a range of mathematical modelling techniques to explore the acid-mediated tumour invasion hypothesis. The models have made a number of predictions which have been experimentally verified. The therapeutic implications, namely either buffering acid or manipulating the phenotypic selection process, have been described. It should be noted that while we have focussed here on the competitive interaction between tumour and normal cells, there is also a cooperative interaction between them in the production of enzymes to degrade extracellular material. We have recently extended the Gatenby and Gawlinski model mentioned in this paper to show that invasion may behave in a biphasic way in response to acid [14], suggesting that more subtle therapeutic approaches may be necessary.

\section{References}

[1] T. Alarcon, H. M. Byrne, P. K. Maini, A cellular automaton model for tumour growth in inhomogeneous environment., J. Theor. Biol. 225 (2003), 257-274.

[2] A. R. A. Anderson, A hybrid mathematical model of solid tumour invasion: the importance of cell adhesion., Math. Med. Biol. 22 (2005), 163-186.

[3] J. Casciari, S. Sotirchos, R. Sutherland, Variations in tumor cell growth rates and metabolism with oxygen concentration, glucose concentration, and extracellular pH, J. Cell. Physiol. 151 (1992), 386-394. 
[4] G. Di Chiro, J. Hatazawa, D. Katz, H. Rizzoli, D. D. Michele, Glucose utilization by intracranial meningiomas as an index of tumor aggressivity and probability of recurrence: a PET study, Radiology 164 (1987), 521-526.

[5] A. Fasano, M. A. Herrero, M. R. Rodrigo, Slow and fast invasion waves in a model of acid-mediated tumour growth, Math. Biosci. 220 (2009), 45-56.

[6] E. Fearon, B. Vogelstein, A genetic model for colorectal tumorigenesis, Cell 61 (1990), 759-767.

[7] I. Fidler, I. Hart, Biological diversity in metastatic neoplasms: origins and implications, Science 217 (1982), 998-1003.

[8] J. Folkman, The role of angiogenesis in tumor growth, Semin. Cancer Biol. 3 (1992), 65-71.

[9] C. M. Friedenreich, M. R. Orenstein, Physical activity and cancer prevention: etiologic evidence and biological mechanisms., J. Nutr. 132 (2002), 3456S-3464S.

[10] R. Gatenby, E. Gawlinski, A reaction-diffusion model of cancer invasion, Cancer Res. 56 (1996), 5745-5753.

[11] R. Gatenby, R. Gillies, Why do cancers have high aerobic glycolysis?, Nature Rev. Cancer 4 (2004), 891-899.

12] G. Martin, R. Jain, Noninvasive measurement of interstitial $p H$ profiles in normal and neoplastic tissue using fluorescence ratio imaging microscopy, Cancer Res. 54 (1994), 5670-5674.

[13] N. Martin, E. Gaffney, R. Gatenby, R. Gillies, I. Robey, P. Maini, A mathematical model of tumour and blood ph, submitted.

[14] N. Martin, E. Gaffney, R. Gatenby, P. Maini, Tumour-stromal interactions in acid-mediated invasion: A mathematical model, submitted.

[15] J. Murray, Mathematical Biology I. An Introduction, Springer-Verlag, 2002, 3rd edition.

[16] A. A. Patel, E. T. Gawlinski, S. K. Lemieux, R. A. Gatenby, A cellular automaton model of early tumor growth and invasion., J. Theor. Biol. 213 (2001), 315-331.

[17] P. Schornack, R. Gillies, Contributions of cell metabolism and $\mathrm{H}^{+}$diffusion to the acidic $p H$ of tumors, Neoplasia 5 (2003), 135-145.

[18] K. Smallbone, R. A. Gatenby, R. J. Gillies, P. K. Maini, D. J. Gavaghan, Metabolic changes during carcinogenesis: potential impact on invasiveness., J. Theor. Biol. 244 (2007), 703-713.

[19] K. Smallbone, R. A. Gatenby, P. K. Maini, Mathematical modelling of tumour acidity., J. Theor. Biol. 255 (2008), 106-112.

[20] K. Smallbone, D. Gavaghan, R. Gatenby, P. K. Maini, The role of acidity in solid tumour growth and invasion, J. Theor. Biol. 235 (2005), 476-484.

[21] R. Thomlinson, L. Gray, The histological structure of some human lung cancers and the possible implications for radiotherapy, Br. J. Cancer 9 (1955), 539-549.

[22] O. Warburg, The Metabolism of Tumours, Constable Press, London, 1930. 